\title{
Outdoor IEEE 802.11g Cellular Network Performance
}

\author{
Elena Lopez-Aguilera, Jordi Casademont, Josep Cotrina \\ Wireless Networks Group - Telematics Department \\ Technical University of Catalonia \\ Barcelona, Spain
}

\begin{abstract}
Most studies about the performance of IEEE 802.11 are limited to a single cell environment. Nevertheless, the idea of designing an outdoor cellular network based on WLAN IEEE 802.11 results very attractive, due to the several advantages that this technology presents: the low cost of the equipment, its operation in unlicensed spectrum and its higher data rates.
\end{abstract}

In this paper, we study the possibility of designing an outdoor cellular network based on the IEEE 802.11g standard. We present its performance under different load conditions and compare this behavior with the results obtained in an isolated single cell environment, without co-channel interference.

Finally, going a step further, this paper explores the IEEE 802.11g cellular network performance for different cluster's sizes, as a method to reduce the interference influence on network's performance.

Keywords - Cellular networks, IEEE 802.11, WLAN.

\section{INTRODUCTION}

Since 1997 when the Institute of Electrical and Electronics Engineers (IEEE) defined the first standard IEEE 802.11 for wireless local area networks it has evolved a lot. The former IEEE 802.11 worked at $2.4 \mathrm{GHz}$ and at data rates of 1 and 2 Mbps. Later it appeared IEEE $802.11 \mathrm{~b}$ that using the same frequency got 11 Mbps. IEEE 802.11a was developed next, this one changed its working frequency to $5 \mathrm{GHz}$ reaching 54 Mbps, but the change of frequency represented a drawback on interoperability with older equipment. In this way, the IEEE $802.11 \mathrm{~g}$ was developed, reaching $54 \mathrm{Mbps}$ but working again at $2.4 \mathrm{GHz}$. Finally, in September 2003 a new working group has begun to work in order to develop IEEE $802.11 \mathrm{n}$ that should get $100 \mathrm{Mbps}$. All these standards' working procedures are practically the same, and only change the modulation, some fields of the physical layer and the duration of the slot and the interframe space times (DIFS, SIFS, PIFS).

Up to now, several papers have been written on different aspects of IEEE 802.11. Reference [1] shows simulation and mathematical results of the throughput of a IEEE 802.11 single cell WLAN, and also propose dynamic adjustments of the backoff algorithm to improve the whole performance. In [2] - [5] we can find several analysis on propagation issues in outdoor environments. All these analysis are based on system traffic saturation, and calculate the saturation throughput. More recently, several papers have appeared that work without this premise and consider situations of no congestion [6]. Finally, the proposals of the working group IEEE 802.11e, that gives Quality of Service (QoS) possibilities to wireless LANs, have also been studied in [7].

A common aspect of all these studies is that they are limited to a single cell environment. However, the idea of designing an outdoor cellular network based on WLAN IEEE 802.11 results very attractive. IEEE 802.11 presents several advantages in front of $2.5 \mathrm{G}$ and $3 \mathrm{G}$ wireless networks, due to the low cost of the equipment required and its operation in unlicensed spectrum. Furthermore, IEEE 802.11 offers higher data rates, far exceeding the maximum data rates offered by EDGE (Enhanced Data Rates for GSM Evolution) and WCDMA (Wideband Code Division Multiple Access) networks.

Having these considerations in mind, the focus of this paper is to evaluate the IEEE 802.11 network performance in a cellular environment; particularly we centre our investigations in IEEE $802.11 \mathrm{~g}$ performance. We present its performance under different load conditions and compare these results with the obtained in a single cell environment.

The organization of the rest of the paper is as follows: section II presents the main topics of the IEEE 802.11 MAC working procedure, section III describes the simulation environment, section IV presents the main results of IEEE $802.11 \mathrm{~g}$ cellular network performance, finally section $\mathrm{V}$ concludes with the most relevant points of the article.

\section{IEEE 802.11 MAC PROTOCOL}

IEEE 802.11 has two operating modes: Distributed Coordination Function (DCF) and Point Coordination Function (PCF). The most common working mode is DCF that uses the medium access control (MAC) algorithm named CSMA/CA (Carrier Sense Multiple Access with Collision Avoidance); it works as follows. Before initiating a transmission, a station senses the channel to determine whether it is busy. If the medium is sensed idle during a period of time named distributed interframe space (DIFS), the station is allowed to transmit. If the medium is sensed busy, the transmission is delayed until the channel is idle again. A slotted binary exponential backoff interval is uniformly chosen 
in $[0, \mathrm{CW}-1]$, where $\mathrm{CW}$ is the contention window. The backoff timer is decreased as long as the channel is sensed idle, stopped when a transmission is in progress, and reactivated when the channel is sensed idle again for more than DIFS. When the backoff timer expires, the station attempts for transmission. After each data frame successfully received, the receiver transmits an acknowledgment frame (ACK) after a short interframe space (SIFS) period. The value of $\mathrm{CW}$ is set to its minimum value, $\mathrm{CW}_{\min }$, in the first transmission attempt, and ascends integer powers of 2 at each retransmission, up to a pre-determined value (usually 1024).

Moreover, the IEEE 802.11 MAC protocol supports two kinds of Basic Service Set (BSS): the independent BSS, known as ad-hoc networks, which have no connection to wired networks, and the infrastructure BSS, which contains an access point (AP) connected to the wired network. The second BSS assimilates to cellular networks with base stations. In this way, we restrict our investigation to infrastructure networks operating in DCF mode.

\section{SIMULATION ENVIRONMENT DESCRIPTION}

In order to analyze the IEEE $802.11 \mathrm{~g}$ performance, we use a simulation tool implemented in UPC (Technical University of Catalonia). Our simulation program, written in $\mathrm{C}++$ programming language, follows all the IEEE 802.11 protocol details. It emulates as closely as possible the real operation of each transmitting station. Our simulation tool permits the IEEE 802.11 protocol emulation in a single cell environment and in a cellular network. On the contrary, the well-known NS-2 Simulator allows the performance evaluation of the IEEE 802.11 only in an isolated cell. In this way, we choose the exposed simulation tool in order to study the cellular network performance.

The simulation tool permits the evaluation of different parameters: throughput (user data correctly transmitted by users without considering retransmissions and headers), average transmission delay, average queue delay, probability of collision, packet error ratio (PER), signal to noise and interference ratio (SIR), proportion of erroneous data packets per data packet received, average number of retransmissions per retransmitted data packet and the fraction of time that the packet reception is interfered with a power higher than the noise power. The simulation tool has been verified comparing the results obtained with the information published in [1], under identical simulation conditions.

The values of the parameters used to obtain the numerical results are exposed in Table I.

The simulation environment consists of 100 hexagonal cells, which form a rectangular area, although only the 36 middle cells are taken to compute the statistics. Each BSS is composed of $1 \mathrm{AP}$ and 10 user stations. Only user stations are transmitting data packets with a constant payload size of 1023 bytes. We consider that data are directed from user stations towards the AP, who forwards them to the infrastructure network. All user stations are under coverage area. Hidden terminal situation is not considered.

We have chosen a cluster of three cells. The adjacentchannel interference is not taken into account.

As path loss model we employ the propagation model for IEEE 802.11 devices operating at $2.4 \mathrm{GHz}$ in outdoor environments specified in [5].

The OFDM has been selected as the modulation scheme for the IEEE 802.11g Extended Rate PHY (ERP-OFDM). It is identical to the modulation scheme employed in the previous IEEE 802.11a PHY, which is very similar to the one chosen in Europe for HIPERLAN/2 PHY. It offers eight PHY modes with different modulation schemes and coding rates; therefore data rates between 6 and $54 \mathrm{Mbps}$ are provided (Table II).

In this paper we assume that the noise over the wireless medium is white Gaussian noise (AWGN). The bit error probability $\left(P_{b}\right)$ depends on the modulation scheme employed. The bit error probability [8] for an $M$-ary QAM modulation with a Gray coding and $M=4,16$, and 64 is calculated by:

$$
P_{b}^{(M)} \approx \frac{1}{\log _{2} M} \cdot\left(1-\left(1-P_{\sqrt{M}}\right)^{2}\right),
$$

where

$$
P_{\sqrt{M}}=2 \cdot\left(1-\frac{1}{\sqrt{M}}\right) \cdot Q\left(\sqrt{\frac{3}{M-1} \cdot \frac{E_{a v}}{N_{o}}}\right) .
$$

$P_{\sqrt{M}}$ is the symbol error probability for the $\sqrt{M}$-ary PAM modulation with the average signal-to-noise per symbol $\frac{E_{a v}}{N_{o}}$.

For BPSK modulation, the bit error probability is the same as the symbol error probability:

$$
P_{b}^{(2)}=Q\left(\sqrt{2 \frac{E_{a v}}{N_{o}}}\right) .
$$

In [9], an upper bound was given on the packet error probability, PER, under the assumption of binary convolutional coding and hard-decision Viterbi decoding with independent errors at the channel input. The PER is obtained following (4):

$$
\text { PER }=1-\left(1-P_{u}^{m}\right)^{\text {bits per interval }} .
$$

The $P_{u}{ }^{m}$ value depends on the PHY mode employed: on its modulation scheme and coding rate. Its detailed calculation is specified in [10].

In order to decide if a packet is received with error at reception time, it is split up in intervals, where the interference power has different values. For each interval the signal to noise and interference ratio is obtained and its correspondent $P_{u}{ }^{m}$ computed.

Then, to decide if a packet is erroneous, for each packet 
interval, a random value between 0 and 1 is calculated. If this value is lower than the PER value, the packet is considered erroneous; otherwise this interval is considered successful and the next one is evaluated in the same way.

\section{SYSTEM BEHAVIOR}

In order to evaluate the IEEE $802.11 \mathrm{~g}$ cellular network behavior, we present its performance in presence of different load conditions. Furthermore, we compare its performance with the results obtained in an isolated single cell environment, without any presence of interfering packets.

TABLE I

MAIN PARAMETERS USED IN THE SIMULATIONS

\begin{tabular}{|l|c|}
\hline & $\begin{array}{c}\mathbf{8 0 2 . 1 1 g} \\
\text { (ERP-OFDM) }\end{array}$ \\
\hline Transmission data rate (Mbps) & $6,9,12,18,24,36,48,54$ \\
\hline MAC header & 34 bytes \\
\hline ACK & 14 bytes \\
\hline PHY Preamble & $16 \mu \mathrm{s}$ \\
\hline PHY Header & $4 \mu \mathrm{s}$ \\
\hline Slot Time & $9 \mu \mathrm{s}$ \\
\hline SIFS & $10 \mu \mathrm{s}$ \\
\hline DIFS & $28 \mu \mathrm{s}$ \\
\hline PIFS & $19 \mu \mathrm{s}$ \\
\hline Minimum backoff window size & 16 \\
\hline Maximum backoff window size & 1024 \\
\hline OFDM symbol interval & $4 \mu \mathrm{s}$ \\
\hline Radio cell & $400 \mathrm{~m}$ \\
\hline Power level at the transmitter output & $30 \mathrm{dBm}$ \\
\hline Noise power & $-96 \mathrm{dBm}$ \\
\hline
\end{tabular}

TABLE II

EIGHT PHY MODES OF IEEE 802.11G ERP-OFDM

\begin{tabular}{|c|c|c|c|}
\hline Mode & Modulation & Code Rate & $\begin{array}{c}\text { Data Rate } \\
\text { (Mbps) }\end{array}$ \\
\hline 1 & BPSK & $1 / 2$ & 6 \\
\hline 2 & BPSK & $3 / 4$ & 9 \\
\hline 3 & QPSK & $1 / 2$ & 12 \\
\hline 4 & QPSK & $3 / 4$ & 18 \\
\hline 5 & $16-\mathrm{QAM}$ & $1 / 2$ & 24 \\
\hline 6 & 16-QAM & $3 / 4$ & 36 \\
\hline 7 & 64-QAM & $2 / 3$ & 48 \\
\hline 8 & 64-QAM & $3 / 4$ & 54 \\
\hline
\end{tabular}

In a cellular network, as the number of packets accessing the medium increases, so does the number of interfering packets. In this way, the fraction of time that the packet reception is interfered with a power higher than the noise power increases with the growth of offered load. Furthermore, the SIR value decreases and consequently the PER rises. In addition, the PER is different for transmission rates employing distinct modulation schemes, in agreement with the bit error probability obtained with (1) - (3).

Fig. 1 shows the average throughput per cell versus average offered load per cell, for different transmission rates. If we compare the throughput performance in a cellular environment with its behavior in a single cell environment, we observe that up to transmission rates of $36 \mathrm{Mbps}$ the presence of interfering packets do not decrease the throughput. Otherwise, for data rates higher than $36 \mathrm{Mbps}$ this decrease becomes considerably higher.

In addition, taking into account the cellular system, each station performance inside a BSS depends strongly of its relative position to the AP. The data frames generated at stations placed near the limit of the coverage area, arrive at its AP with lower power level. Fig. 2 presents the SIR performance versus average offered load per station for packets generated at stations placed at different distance to the AP inside its BSS, employing a transmission data rate of 48 Mbps. The SIR value for the most distant station decreases a $71.85 \%$ in comparison with the nearest one, as the offered load to the system rises.

This situation provokes an important PER growth as the stations increase their distance to the AP (Fig. 3). Thereby, the throughput performance becomes poorer with the distance increase (Fig. 4). The throughput decreases considerably and becomes closer to zero, as the offered load to the system rises. On the other hand, the nearest stations take advantage of the situation and increase their throughput a $48.7 \%$ at higher offered load, in comparison with its performance in a single cell environment.

The PER is higher as the transmission data rate increases. In this way, the system performance at higher rates moves away from the obtained in a single cell environment. Employing a data rate of $54 \mathrm{Mbps}$, the distant stations decrease their throughput performance faster, becoming closer to zero even when the system is low loaded (Fig. 5).

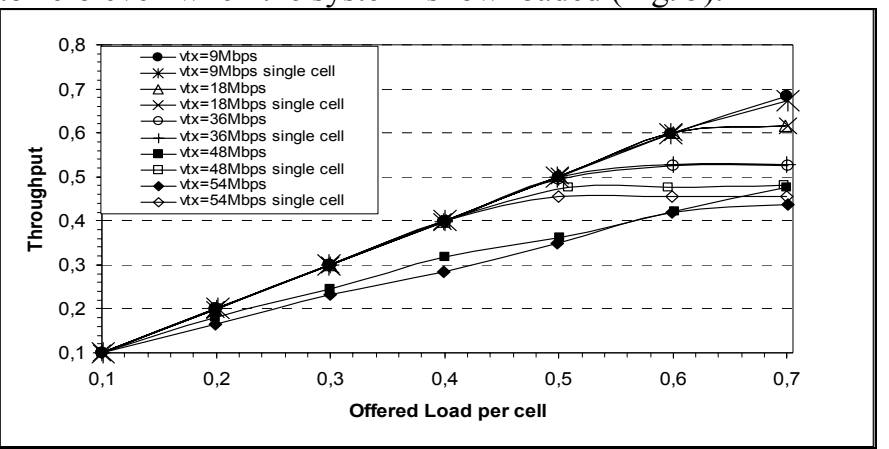

Figure 1. Throughput per cell versus offered load per cell 


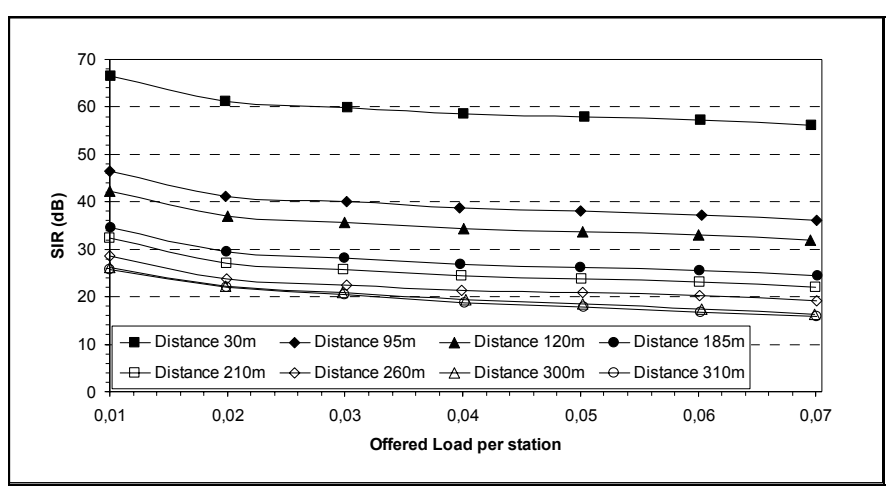

Figure 2. SIR versus offered load per station for $\mathrm{vtx}=48 \mathrm{Mbps}$

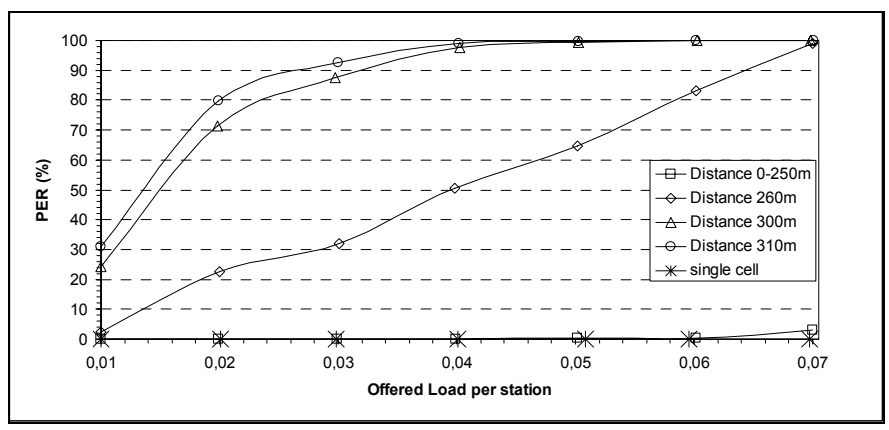

Figure 3. PER versus offered load per station for $v t x=48 \mathrm{Mbps}$

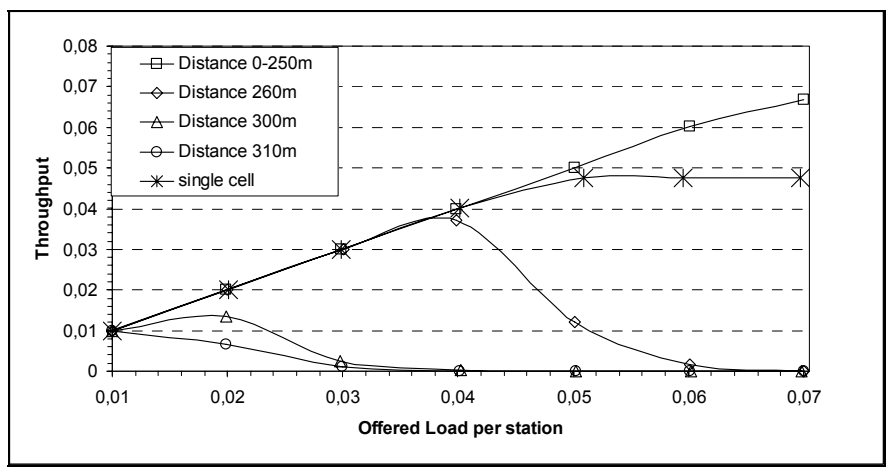

Figure 4. Throughput versus offered load per station for $\mathrm{vtx}=48 \mathrm{Mbps}$

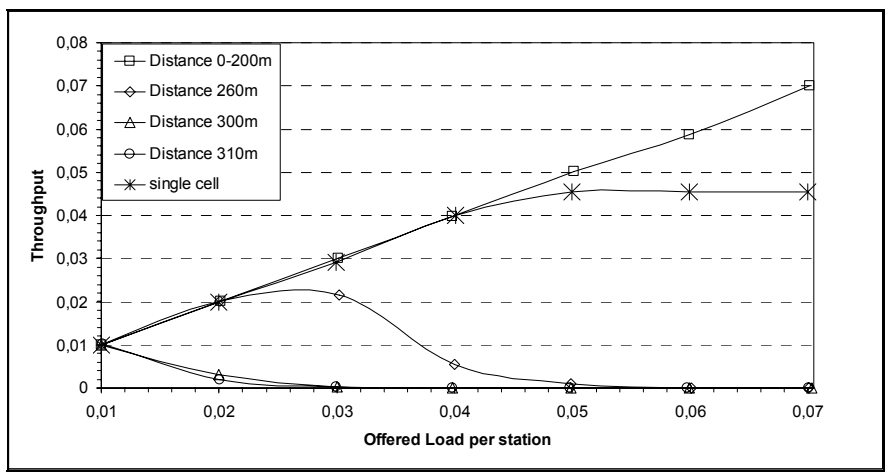

Figure 5. Throughput versus offered load per station for $\mathrm{vtx}=54 \mathrm{Mbps}$

Going a step further, we study the IEEE $802.11 \mathrm{~g}$ cellular network performance when the number of cells that form the cluster changes. The increase in the cluster's size is a standard method to reduce the interference influence on networks performance.

The fraction of time that the packet reception is interfered with a power higher than the noise power decreases with the cluster's value. In this way, the SIR (Fig. 6) increases with the cluster's value, and its performance comes near to the presented in a single cell environment. Consequently, the PER (Fig. 7) decreases considerably and becomes closer to zero when the number of cells forming the cluster is seven, employing a transmission data rate of $48 \mathrm{Mbps}$. Thereby, their value comes closer to the obtained in a single cell environment.

In this way, as the cluster's size increases, the throughput performance for the different mobile stations comes near to the obtained in a single cell environment, employing a transmission data rate of $48 \mathrm{Mbps}$ (Fig. 8).

On the other hand, employing a data rate of $54 \mathrm{Mbps}$ and a cluster size of seven cells, the throughput for the most distant stations improves its performance, but it still becomes distant from the obtained in a single cell environment, as the offered load to the system rises (Fig. 9).

In a cellular environment, the SIR is proportional to the number of cells forming the cluster. The worse situation is for a user station placed at the edge of a cell located in the center of the network, in presence of six interfering cells. In this case, the SIR follows approximately (5):

$$
\operatorname{SIR} \approx \frac{1}{6} \cdot(\sqrt{3 K})^{\gamma},
$$

where $K$ is the cluster size and $\gamma$ is the propagation factor employed.

Following the propagation model specified in [5], $\gamma$ takes a value of 4 . In this way, the SIR value is proportional to $K^{2}$. Consequently, the maximum SIR increase between a network employing a cluster of three cells and another using one of four is $2.5 \mathrm{~dB}$, between one with cluster's size of three cells and other with seven cells is $7.36 \mathrm{~dB}$, and finally between a network using a cluster of four cells and another employing one of seven is $4.86 \mathrm{~dB}$. The results presented in Fig. 6 agree with the maximum values exposed.

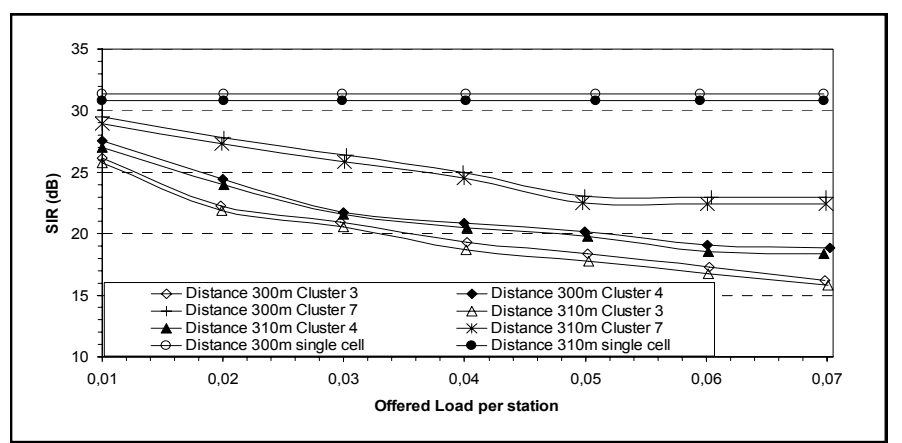

Figure 6. SIR versus offered load per station, for different clusters and $\mathrm{vtx}=48 \mathrm{Mbps}$ 


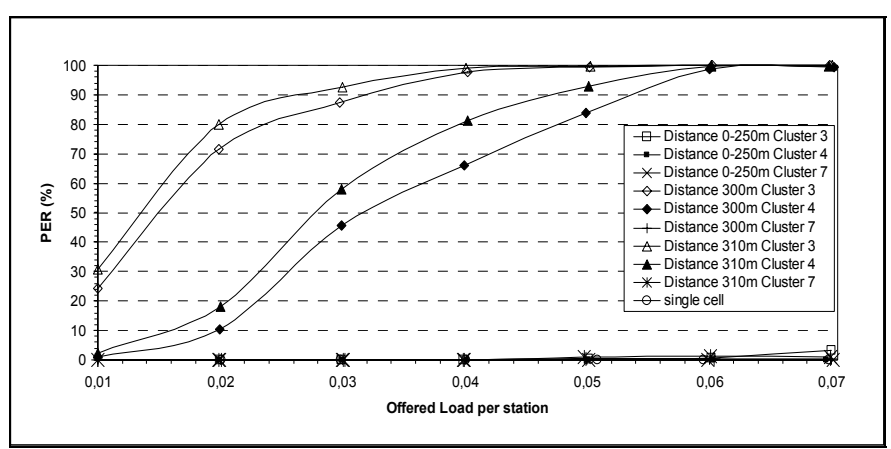

Figure 7. PER versus offered load per sation, for different clusters and $v t x=48 \mathrm{Mbps}$

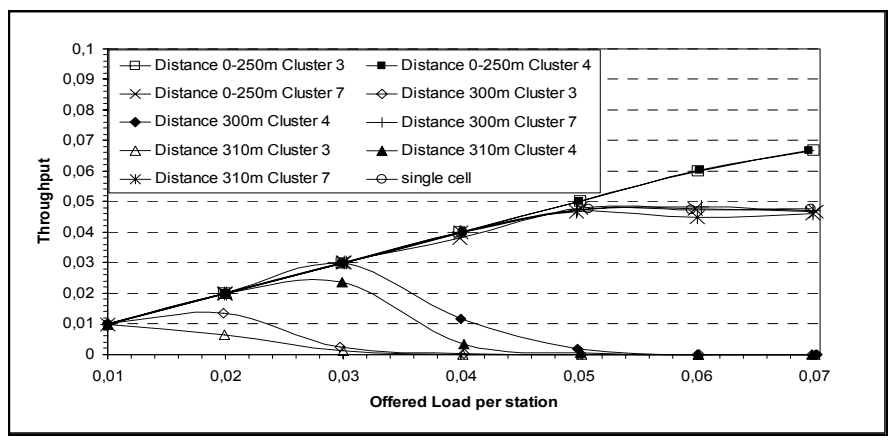

Figure 8. Throughput versus offered load per station, for different clusters and $v t x=48 \mathrm{Mbps}$

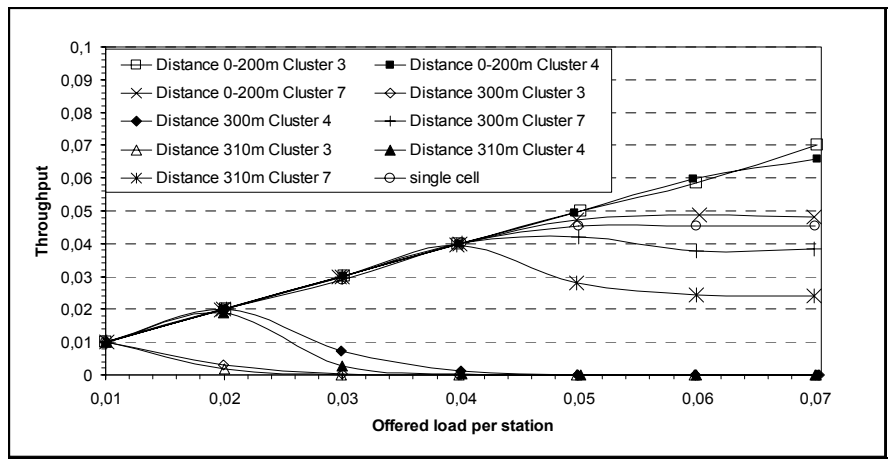

Figure 9. Throughput versus offered load per station, for different clusters and $\mathrm{vtx}=54 \mathrm{Mbps}$

\section{CONCLUSIONS}

This paper presents a study of the IEEE 802.11 network performance in an outdoor cellular environment; particularly we centre our investigations in IEEE $802.11 \mathrm{~g}$ performance.

Up to now, most studies about the performance of IEEE 802.11 are focused on a single cell environment. This scenario is realistic when the standard operates in small isolated areas.

However, due to the several advantages presented by IEEE 802.11 networks, the idea of designing a cellular network becomes very attractive.

Thereby, in this paper we expose the outdoor IEEE $802.11 \mathrm{~g}$ cellular network performance, taking into account the influence of interfering packets. The results have been evaluated in relation with the obtained in an isolated single cell environment, without co-channel interference. Each station performance inside a BSS depends strongly of its relative position to the AP. Thereby, the throughput performance becomes poorer with the distance increase.

Finally, this paper studies the IEEE $802.11 \mathrm{~g}$ cellular network performance for different cluster's sizes. It supposes a method to reduce the interference influence on network's performance. When the number of cells forming the cluster is seven, the interference influence is reduced considerably and the cellular network's performance comes near the single cell environment behavior, employing a transmission data rate of 48 Mbps. When the system is working at faster data rates, higher cluster's sizes are necessary, in order to assimilate the system performance to the obtained in a single cell environment.

Actually, at $2.4 \mathrm{GHz}$ it is only allowed to work with three non-overlapped channels. Thereby, the employment of cluster's sizes of four and seven cells is not possible taking into account the actual legislation. On the other hand, the legislation at $5 \mathrm{GHz}$ allows the employment of cluster's sizes of four and seven cells.

\section{ACKNOWLEDGMENT}

This research has been funded by FEDER and the Spanish Government through CICYT project TIC2003-01748.

\section{REFERENCES}

[1] G. Bianchi, "Performance analysis of the IEEE 802.11 Distributed Coordination Function", IEEE Journal on selected areas in communications, Vol. 18, No. 3, March 2000, pp. 535 - 547.

[2] M. V. Clark, K. K. Leung, B. McNair, Z. Kostic, "Outdoor IEEE 802.11 cellular networks: radio link performance", IEEE International Conference on Communications 2002 (ICC 2002), Vol. 1, April 2002, pp. 512 - 516.

[3] K. K. Leung, B. McNair, L. J. Cimini, J. H. Winters, "Outdoor IEEE 802.11 cellular networks: MAC protocol design and performance", IEEE International Conference on Communications 2002 (ICC 2002), Vol. 1, April 2002, pp. 595 - 599.

[4] C. Steger, P. Radosavljevic, J. P. Frantz, "Performance of IEEE $802.11 \mathrm{~b}$ wireless LAN in an emulated mobile channel", Vehicular Technology Conference 2003. (VTC 2003-Spring), Vol. 2, April 2003, pp. 1479 - 1483.

[5] D. B. Green, M. S. Obaidat, "An accurate line of sight propagation performance model for ad-hoc 802.11 wireless LAN (WLAN) devices", International Conference on Communications 2002 (ICC 2002), Vol. 5, April 2002, pp. $3424-3428$.

[6] E. Ziouva, T. Antonakopoulos, "The IEEE 802.11 Distributed Coordination Function in small-scale ad-hoc wireless LANs", International Journal of wireless information networks, Kluwer Academic Publishers, Vol. 10, No. 1, January 2003, pp. 1 - 15.

[7] S. Choi, J. del Prado, S. Shankar N, S. Mangold, "IEEE 802.11e contention-based channel access (EDCF) performance evaluation," IEEE International Conference on Communications 2003 (ICC 2003), Vol. 2, May 2003, pp. $1151-1156$.

[8] J. G. Proakis, Digital Communications, $4^{\text {th }}$ ed., McGraw Hill, New York, 2001.

[9] M. B. Pursley, D. J. Taipale, "Error probabilities for spread-spectrum packet radio with convolutional codes and Viterbi decoding", IEEE Transactions on communications, Vol. COM-35, No. 1, Jan 1987, pp. 1-12.

[10] D. Qiao, S. Choi, K. G. Shin, "Goodput analysis and link adaptation for IEEE 802.11a wireless LANs", IEEE Transactions on mobile computing, Vol. 1, No. 4, October - December 2002, pp. 278 - 292. 